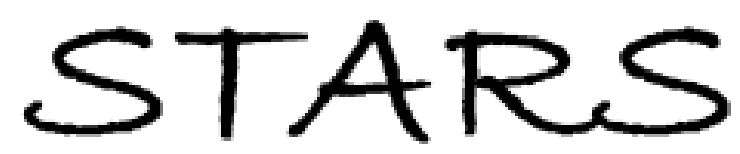

University of Central Florida

STARS

Faculty Bibliography 1990s

Faculty Bibliography

$1-1-1999$

\title{
Statistical estimation of locations of lightning events
}

Marianna Pensky

University of Central Florida

John R. Cannon

University of Central Florida

Find similar works at: https://stars.library.ucf.edu/facultybib1990

University of Central Florida Libraries http://library.ucf.edu

This Article is brought to you for free and open access by the Faculty Bibliography at STARS. It has been accepted for inclusion in Faculty Bibliography 1990s by an authorized administrator of STARS. For more information, please contactSTARS@ucf.edu.

\section{Recommended Citation}

Pensky, Marianna and Cannon, John R., "Statistical estimation of locations of lightning events" (1999).

Faculty Bibliography 1990s. 2783.

https://stars.library.ucf.edu/facultybib1990/2783

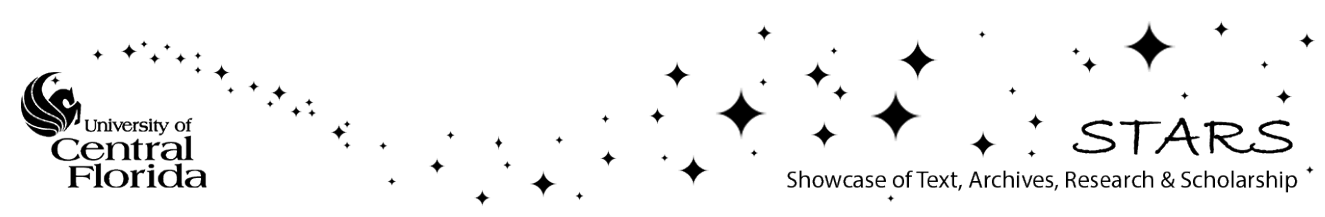




\title{
Statistical estimation of locations of lightning events
}

\author{
Marianna Pensky and John R. Cannon \\ Department of Mathematics, University of Central Florida, Orlando, Florida
}

\begin{abstract}
In this paper, a statistical approach to the retrieval of lightning locations is proposed for the first time. This novel approach views the errors of the time measurements as random variables rather than unknown numbers. The unknown location $(x, y, z)$ as well as the standard deviation $\sigma$ of the errors are treated as unknown parameters of a statistical model and are estimated using the maximum likelihood estimation (MLE) technique. On the basis of Monte Carlo simulations these statistical estimators are compared with the least squares estimators (LSE), as well as the solutions of the system of linear equations proposed by Koshak and Solakiewicz [1996]. Although the method is general, the Lightning Detection and Ranging (LDAR) system currently used at the Kennedy Space Center is chosen as a model for simulations. Simulations show that the MLE always gives better precision than the LSE technique. Also, it is demonstrated that if the time measurements are fairly accurate and a thunderstorm takes place in the neighborhood of the measuring sites (the distance is less than $80 \mathrm{~km}$ ), the MLE significantly improves the accuracy of the solutions of the system of linear equations.
\end{abstract}

\section{Introduction}

The problem of detection of locations of lightning events on the basis of ground-based measurements has been studied extensively within the last three decades [see, e.g., Cianos et al., 1972; Hager and Wang, 1995; Koshak and Solakiewicz, 1996; Lee, 1986; Lewis et al., 1960; MacClement and Murty, 1978; Murty and MacClement, 1973; Panyukov and Strauss, 1996; Proctor, 1971; Rustan et al., 1980; Taylor, 1978; Thompson et al., 1994]. All of the authors derive the location of a lightning event from the times of arrival of electromagnetic radiation at several locations. The differences in the times of arrival are converted into differences in distances from the point of origin $(x, y, z)$ of the radiation to $(m+1)$ receiving sites located in $x y$ plane at $\left(a_{i}, b_{i}, c_{i}\right), i=0, \ldots, m$. Usually, site 0 is chosen to be the origin $(0,0,0)$ of a local rectangular coordinate system, and we will follow this practice.

Denoting the time of arrival of the signal at the site $i$ by $t_{1}$, $i=0, \ldots, m$, and the distance from the point of origin of the signal $(x, y, z)$ to the site $i$ by

$$
d_{i}=\sqrt{\left(x-a_{i}\right)^{2}+\left(y-b_{i}\right)^{2}+\left(z-c_{i}\right)^{2}}
$$

we see that

$$
d_{\imath}-d_{0}=c\left(t_{\imath}-t_{0}\right), \quad i=1, \ldots, m,
$$

where $c=299,792 \mathrm{~km} / \mathrm{s}$ is the measured speed of light. Current practice is to use site 0 as the trigger for the initiation of a time of arrival measurement at all sites. Transmission times to and from the other sites are taken into account in the

Copyright 1999 by the American Geophysical Union.

Paper number 1998JD200111.

0148-0227/99/1998JD200111\$09.00 determination of the $t_{i}, i=1, \ldots, m$. Thus

$$
u_{i}=c\left(t_{i}-t_{0}\right), \quad i=1, \ldots, m,
$$

are calculated, and the system of $\boldsymbol{m}$ equations

$$
\begin{gathered}
\sqrt{\left(x-a_{i}\right)^{2}+\left(y-b_{i}\right)^{2}+\left(z-c_{i}\right)^{2}}-\sqrt{x^{2}+y^{2}+z^{2}}=u_{i}, \\
i=1, \ldots, m
\end{gathered}
$$

has to be solved. Here $a_{0}=b_{0}=c_{0}=0$.

A variety of techniques has been used for determination of the lightning locations $(x, y, z)$ from the arrival times. The differences between the studies include the number of measuring sites, various approaches to the solution of the nonlinear system of equation (3), as well as different treatment of the solution error. From a mathematical point of view, the vast majority of methods of retrieval of lightning locations fall into one of the categories discussed in this section.

The first cluster of methods views the three-dimensional source location $(x, y, z)$ as the mathematical intersection of hyperboloids of two sheets [see, e.g., Proctor, 1971; Thompson et al, 1994]. These methods usually involve a solution of a nonlinear system of equations. The second class of techniques is based on a least squares estimator which minimizes the squared sum of differences between the left and the right side in equation (3) [see Panyukov and Strauss, 1996]. Computationally, it leads to global minimization of the function

$$
\begin{array}{r}
F(x, y, z)=\sum_{i=1}^{m}\left[\sqrt{\left(x-a_{i}\right)^{2}+\left(y-b_{i}\right)^{2}+\left(z-c_{i}\right)^{2}}\right. \\
\left.-\sqrt{x^{2}+y^{2}+z^{2}}-c\left(t_{i}-t_{0}\right)\right]^{2} .
\end{array}
$$


The third class of approaches is based on linearization of the system of equation (3) and subsequent solution of the system of linear equations [see Cannon, 1995; Koshak and Solakiewicz, 1996]. Although equation (3) appears to be nonlinear at first glance, system (3) can be easily linearized. For instance, in the case when all measuring sites are located on a plane (i.e., $\left.c_{2}=0, i=1,2, \ldots, m\right)$, introduction of the new variables

$$
d_{0}=r=\sqrt{x^{2}+y^{2}+z^{2}}, \quad r_{i}=\sqrt{a_{i}^{2}+b_{i}^{2}},
$$

and simple algebraic transformations result in the system of equations

$$
\sqrt{r^{2}+r_{i}^{2}-2 a_{i} x-2 b_{2} y}-r=u_{i}, \quad i=1, \ldots, m .
$$

Shifting $r$ to the right side of equation (6) and squaring both sides, we find that $x, y, r$ satisfy the system of linear equations

$$
a_{2} x+b_{2} y+u_{i} r=\left(r_{2}^{2}-u_{i}^{2}\right) / 2, \quad i=1, \ldots, m .
$$

As the solution $(x, y, r)$ of system (7) is obtained, the height $z$ is evaluated as

$$
z=\sqrt{r^{2}-x^{2}-y^{2}}
$$

Some efforts have also been made to investigate the errors of the solutions [see, e.g., Koshak and Solakiewicz, 1996]. Analysis was done from a deterministic point of view treating the errors in the observations as unknown variables that influence the precision of the solution.

In what follows, we propose a comprehensive statistical approach to the retrieval of lightning locations. To the best of our knowledge, this is the first statistical formulation of the problem. This approach views the errors of the time measurements as random variables rather than unknown numbers. The unknown location $(x, y, z)$ as well as the standard deviation $\sigma$ of the errors are treated as unknown parameters of a statistical model and are estimated using the maximum likelihood estimation (MLE) technique [see Lehmann, 1983]. These statistical estimators are compared with the solutions of the system of linear equation (7), as well as the least squares estimators (LSE) on the basis of Monte Carlo simulations [see Buslenko et al.\}, 1966]. Thus the present paper has two objectives: (1) introduction of a new statistical approach to estimation of the lightning locations, and (2) application of statistical simulations to evaluate existing methods.

In section 2 of the present paper, we describe the statistical model developed to retrieve the unknown lightning locations and the construction of the (MLE) of $(x, y, z)$ and $\sigma$. We also discuss the numerical implementation of the MLE and the choice of the initial values in the evaluation process. In section 3, we use the Monte Carlo simulations for investigation of the merits and the defects of the three methods: the MLE, the LSE, and the solution of the system of algebraic equations. In section 4 , the advantages and disadvantages of these methods are discussed and some recommendations are made for their comparative application. Although the method is general, the Lightning Detection and Ranging (LDAR) system currently used at the Kennedy Space Center is chosen as a model for simulations.

\section{The Maximum Likelihood Estimator of the Lightning Location}

Let us consider calculating the locations of lightning events using a statistical formulation. We assume that times $t_{\imath}$ of arrival of the signal at the site $i$ are measured with independent normally distributed errors $\varepsilon_{2} \sim N\left(0, \rho^{2}\right)$ and $i=0,1, \ldots, m$, where $\rho$ is the unknown standard deviation of the errors in time measurements. Denote $\sigma=c \rho$, the standard deviation of the distance measurements $c t_{i}$, $i=0,1, \ldots, m$. Introducing a vector $U=\left(u_{1}, u_{2}, \ldots, u_{m}\right)^{T}$, we see that

$$
u_{i}=c\left(t_{i}-t_{0}\right)+c\left(\varepsilon_{i}-\varepsilon_{0}\right) \equiv \mu_{2}+c\left(\varepsilon_{i}-\varepsilon_{0}\right),
$$

where $\mu_{2}$ is the $i$ th component of $\mu$, the vector function of the unknown parameters $x, y, z$ :

$$
\begin{aligned}
\mu_{i}=\mu_{i}(x, y, z)= & \sqrt{\left(x-a_{i}\right)^{2}+\left(y-b_{i}\right)^{2}+\left(z-c_{i}\right)^{2}} \\
& -\sqrt{x^{2}+y^{2}+z^{2}}, \quad i=1, \ldots, m .
\end{aligned}
$$

It is easy to notice that the first components $\mu_{i}$ in equation (9) are deterministic, while $c\left(\varepsilon_{i}-\varepsilon_{0}\right)$ are normally distributed with zero expectations, variances $\operatorname{var}\left[c\left(\varepsilon_{\imath}-\varepsilon_{0}\right)\right]=2 \sigma^{2}$ and covariances $\operatorname{cov}\left[c\left(\varepsilon_{2}-\varepsilon_{0}\right), c\left(\varepsilon_{j}-\varepsilon_{0}\right)\right]=\sigma^{2}$ when $i \neq j$. Therefore, the vector $U$ has the $m$-dimensional normal distribution with the mean $\mu$ and the covariance matrix $\sum=\sigma^{2} B$

$$
\begin{aligned}
& p\left(U \mid x, y, z, \sigma^{2}\right) \\
& =\frac{\exp \left\{-0.5 \sigma^{-2}[U-\mu(x, y, z)]^{T} B^{-1}[U-\mu(x, y, z)]\right\}}{(2 \pi)^{m / 2} \sigma^{m} \sqrt{\operatorname{det}(B)}}
\end{aligned}
$$

where the matrix $B$ has the form

$$
B_{i j}= \begin{cases}1, & \text { if } i \neq j \\ 2, & \text { if } i=j\end{cases}
$$

Note that since $c$ is very large, the value of $\sigma$ is not negligibly small, and hence the stochastic nature of the problem cannot be ignored.

The MLE is the value of $x, y, z, \sigma$ maximizing the logarithm of the likelihood function

$$
\begin{aligned}
& L\left(x, y, z, \sigma^{2} \mid U\right) \\
& =-m \ln \sigma-0.5 \sigma^{-2}[U-\mu(x, y, z)]^{T} B^{-1}[U-\mu(x, y, z)]
\end{aligned}
$$

given an observation on the vector $U$. Taking the derivatives of (13) with respect to $\sigma$, and equating it to zero, one realizes that the maximum of the function (13) is attained at the point $(\widehat{x}, \widehat{y}, \widehat{z}, \widehat{\sigma})$ such that $(\widehat{x}, \widehat{y}, \widehat{z})$ is the global minimum of the function

$$
Q(x, y, z)=[U-\mu(x, y, z)]^{T} B^{-1}[U-\mu(x, y, z)]
$$

and

$$
\widehat{\sigma}=m^{-1 / 2} \sqrt{[U-\mu(\widehat{x}, \widehat{y}, \widehat{z})]^{T} B^{-1}[U-\mu(\widehat{x}, \widehat{y}, \widehat{z})]} .
$$

It is easy to verify that $Q(x, y, z) \rightarrow 0$ as $|x| \rightarrow \infty$, $|y| \rightarrow \infty$, and $|z| \rightarrow \infty$, which creates complications in the process of finding a global minimum of the function (14): 
The optimization procedure is "tempted" to offer a point "infinitely far away" from zero as a global minimum of $Q(x, y, z)$. To avoid this situation we minimize the function

$$
Q^{*}(x, y, z)= \begin{cases}Q(x, y, z), & \text { if } x^{2}+y^{2}+z^{2} \leq R^{2}, \\ R, & \text { if } x^{2}+y^{2}+z^{2}>R^{2},\end{cases}
$$

where we choose $R=1000$. The above choice of $R$ means that we are searching for a lightning location inside the circle with the center at the site 0 and the radius $1000 \mathrm{~km}$, and that we are not interested in any solutions outside this area.

Powell's direction set method [see Press et al., 1986, Section 10.5] was chosen for minimization of the function (16). Since the solution of the linear system (7) provides a very fast algorithm for estimation of the location of the lightning, this linear solution was used as the initial point in computation of a global minimum of function (16).

\section{Comparison of Methods via Monte Carlo Simulation}

The Monte Carlo simulations were used to examine the performance of the MLE of the lightning locations and to compare it with the performances of the LSE and of the estimator based on the solution of the system of linear equations (SSLE). The application of Monte Carlo simulations instead of the use of real LDAR data was inspired by the following considerations. First, with the simulated data, the exact "true location" of the lightning event is always known, which makes the comparison much more precise. Second, the Monte Carlo simulations allow one to generate any amount of data. For example, for the present paper, as many as 30,000 lightning events were generated at various locations. Third, the lightning events can be generated at the locations of choice which enables one to study the estimators from different points of view. All these show that the comparison based on the simulated data is much more flexible and thorough than the comparison based on real LDAR data.

To be more specific, for our simulations, we chose the LDAR system currently used at the Kennedy Space Center. This system contains seven measuring sites located at the tops of antennas. The origin $(0,0,0)$ is located at the top of the antenna at the site 0 , and the heights of the antennas at the other sites are adjusted taking the curvature of the earth into account to be nearly in the $x y$ plane; that is, $c_{i}=0$, $i=0,1, \ldots, m$, and $m=6$.

For the sake of comparison of MLE, LSE, and SSLE, $n$ lightning events were generated at random locations. For the $j$ th lightning event, the distances $d_{i, j}, i=0, \ldots, 6$, $j=1, \ldots, n$, between the lightning location $\left(x_{j}, y_{j}, z_{j}\right)$ and the seven sites $\left(a_{i}, b_{i}, 0\right)$, were calculated (see equation (1)). Then the independent normally distributed errors $\varepsilon_{\imath, j} \sim N\left(0, \sigma^{2}\right)$, $i=0, \ldots, 6, j=1, \ldots, n$, were added to each of the measurements, and the observations $u_{i, j}=\left(d_{i, j}+\varepsilon_{i, j}\right)-$ $\left(d_{0, j}+\varepsilon_{0, j}\right)$ were formed. On the basis of the observations $u_{\imath, j}, i=1, \ldots, 6, j=1, \ldots, n$, three estimators $\left(\widehat{x}_{j}, \widehat{y}_{j}, \widehat{z}_{j}\right)$ of the location were calculated: the LSE which minimizes function (4), the SSLE based on the solution of a system of linear equation (7), and the MLE estimator described in section 2. For both the LSE and MLE, the SSLE was chosen as the initial point for starting the iterations. Then the mean squared errors and the relative errors

$$
\begin{aligned}
\Delta_{\jmath}^{2} & =\left(\widehat{x}_{j}-x_{j}\right)^{2}+\left(\widehat{y}_{j}-y_{j}\right)^{2}+\left(\widehat{z}_{j}-z_{j}\right)^{2}, \\
\delta_{\jmath} & =\sqrt{\frac{\left(\widehat{x}_{j}-x_{\jmath}\right)^{2}+\left(\widehat{y}_{j}-y_{j}\right)^{2}+\left(\widehat{z}_{j}-z_{j}\right)^{2}}{x_{j}^{2}+y_{j}^{2}+z_{j}^{2}}},
\end{aligned}
$$

respectively, were evaluated for each of the three estimation methods. After the process of generation of lightning events was repeated $n$ times, the sample standard deviation and the average relative error

$$
\Delta=\sqrt{n-1 \sum_{j=1}^{n} \Delta_{j}^{2}}, \quad \delta=n^{-1} \sum_{j=1}^{n} \delta_{j},
$$

respectively, were calculated. Then the sample standard deviation $\Delta$ and the average relative error $\delta$ were used as characteristics of the precision LSE, MLE and SSLE.

The results of the simulation are summarized in the tables and figures. Tables 1 and 2 show the sample standard deviations when the lightnings occur at various distances from site 0 (the origin). All distances are measured in kilometers. Each line of the table represents the simulations of lightning events at a fixed horizontal distance $D$ from the origin. For each value of $D$, we performed $n=100$ simulations of lightning events at the points $\left(x_{j}, y_{j}, z_{j}\right)$, where $x_{j}=D \cos \left(\alpha_{j}\right), y_{j}=$ $D \sin \left(\alpha_{j}\right)$, with $\alpha$, being uniformly distributed in the interval $(0,2 \pi)$, and $z_{j}$ being uniformly distributed in the interval $(0,20 \mathrm{~km})$. The first column of both tables represents the distance $D$ from the origin; the second, third and fourth columns give the values of $\Delta$ for the LSE, MLE, and SSLE, respectively. The last column contains the estimators of $\sigma$

$$
\widehat{\sigma}=\sqrt{n^{-1} \sum_{j=1}^{n} \widehat{\sigma}_{j}^{2}},
$$

where $\widehat{\sigma}_{j}$ is the estimator of $\sigma$ based on the $j$ th lightning event,

Table 1. The Values of $\Delta$

\begin{tabular}{rrccc}
\hline$D, \mathrm{~km}$ & $\Delta$ LSE, $\mathrm{km}$ & $\Delta \mathrm{MLE}, \mathrm{km}$ & $\Delta$ SSLE, $\mathrm{km}$ & $\widehat{\sigma}$ \\
\hline 5.0 & 0.4397 & 0.4105 & 0.4406 & 0.0538 \\
10.0 & 0.4982 & 0.4416 & 0.5585 & 0.0496 \\
15.0 & 0.7830 & 0.6781 & 0.8415 & 0.0481 \\
20.0 & 1.3609 & 1.0939 & 1.3844 & 0.0515 \\
25.0 & 1.6475 & 1.2490 & 1.7693 & 0.0494 \\
30.0 & 2.2973 & 1.8338 & 2.3456 & 0.0483 \\
35.0 & 2.9924 & 2.2530 & 3.0861 & 0.0527 \\
40.0 & 3.7547 & 2.7311 & 3.7925 & 0.0524 \\
45.0 & 5.2538 & 3.7108 & 5.0168 & 0.0489 \\
50.0 & 5.4974 & 3.8688 & 5.2539 & 0.0467 \\
55.0 & 7.9000 & 5.4198 & 7.5596 & 0.0498 \\
60.0 & 9.4728 & 6.1798 & 8.6802 & 0.0531 \\
65.0 & 8.5223 & 6.0295 & 8.0401 & 0.0535 \\
70.0 & 14.5451 & 8.7364 & 12.7399 & 0.0496 \\
75.0 & 21.0432 & 11.1690 & 13.6035 & 0.0524 \\
80.0 & 15.8921 & 11.3057 & 14.4006 & 0.0501 \\
85.0 & 20.4448 & 18.9204 & 17.3037 & 0.0483 \\
90.0 & 19.7563 & 12.3624 & 15.4568 & 0.0493 \\
95.0 & 28.5680 & 16.7671 & 20.5788 & 0.0529 \\
100.0 & 33.2571 & 19.7025 & 21.1299 & 0.0522 \\
\hline
\end{tabular}

Here $\sigma=0.05 \mathrm{~km}$. 
Table 2. The Values of $\Delta$

\begin{tabular}{rrrrc}
\hline$D, \mathrm{~km}$ & $\Delta \mathrm{LSE}, \mathrm{km}$ & $\Delta \mathrm{MLE}, \mathrm{km}$ & $\Delta \mathrm{SSLE}, \mathrm{km}$ & $\hat{\sigma}$ \\
\hline 5.0 & 0.8788 & 0.8182 & 0.8753 & 0.1077 \\
10.0 & 0.9987 & 0.8857 & 1.0614 & 0.0996 \\
15.0 & 1.5743 & 1.3552 & 1.6683 & 0.0963 \\
20.0 & 2.8471 & 2.1876 & 2.7931 & 0.1035 \\
25.0 & 3.3800 & 2.4240 & 3.4452 & 0.0989 \\
30.0 & 4.8021 & 3.7930 & 4.5917 & 0.0970 \\
35.0 & 6.1489 & 4.6028 & 5.9469 & 0.1058 \\
40.0 & 7.4446 & 5.3379 & 6.9998 & 0.1048 \\
45.0 & 13.4676 & 7.8738 & 8.8508 & 0.0978 \\
50.0 & 12.8694 & 8.2602 & 10.3516 & 0.0936 \\
55.0 & 20.6936 & 12.8799 & 14.1024 & 0.0998 \\
60.0 & 27.3042 & 13.4513 & 16.1513 & 0.1069 \\
65.0 & 20.0867 & 12.3939 & 14.0976 & 0.1071 \\
70.0 & 97.7677 & 28.7038 & 23.1457 & 0.1001 \\
75.0 & 156.2394 & 31.7085 & 25.7241 & 0.1059 \\
80.0 & 47.8416 & 32.3223 & 27.7152 & 0.1010 \\
85.0 & 117.7909 & 94.9241 & 39.4569 & 0.0976 \\
90.0 & 113.3804 & 28.4990 & 26.3727 & 0.0997 \\
95.0 & 179.8835 & 67.7110 & 34.5434 & 0.1069 \\
100.0 & 184.2410 & 88.3532 & 43.0049 & 0.1052 \\
\hline
\end{tabular}

Here $\sigma=0.01 \mathrm{~km}$.

$j=1, \ldots, n$ (see equation (15)). Tables 1 and 2 represent simulation results when $\sigma=0.05 \mathrm{~km}$ and $\sigma=0.1 \mathrm{~km}$, respectively, which correspond to the standard deviations of the time measurements $\varrho=0.05 / c \simeq 166.8 \times 10^{-9}$ and $\varrho=0.1 / c=\simeq 333.6 \times 10^{-9}$. Here, $c=299,792 \mathrm{~km} / \mathrm{s}$ is the measured speed of light. Table 3 contains the results of simulations for various values of $\sigma$, when the thunderstorm takes place in the neighborhood of the receiving sites, namely, when lightning events are uniformly distributed in the parallelepiped $[-40,40] \times[-40,40] \times[0,20]$.

Table 4 studies the relative performance of the LSE, MLE, and SSLE in terms of azimuth. Each of the lines of the table

Table 3. The Values of $\Delta$ for Various Values of $\sigma$

\begin{tabular}{crrrc}
\hline$D, \mathrm{~km}$ & $\Delta \mathrm{LSE}, \mathrm{km}$ & $\Delta \mathrm{MLE}, \mathrm{km}$ & $\Delta \mathrm{SSLE}, \mathrm{km}$ & $\widehat{\sigma}$ \\
\hline 0.01 & 0.7731 & 0.5692 & 0.7990 & 0.0100 \\
0.02 & 1.2657 & 1.0032 & 1.2983 & 0.0204 \\
0.03 & 1.7718 & 1.3305 & 1.7758 & 0.0296 \\
0.04 & 2.3696 & 1.6676 & 2.4141 & 0.0396 \\
0.05 & 2.8055 & 2.0830 & 2.7699 & 0.0520 \\
0.06 & 3.8693 & 2.5121 & 3.7992 & 0.0586 \\
0.07 & 4.2254 & 3.3249 & 3.9700 & 0.0687 \\
0.08 & 5.7234 & 3.6117 & 5.4302 & 0.0887 \\
0.09 & 5.3531 & 3.5847 & 4.4084 & 0.0892 \\
0.10 & 7.4095 & 4.0224 & 5.0496 & 0.0937 \\
0.11 & 7.4846 & 5.2593 & 6.8898 & 0.1061 \\
0.12 & 8.8483 & 6.1101 & 7.6629 & 0.1223 \\
0.13 & 9.9822 & 6.1090 & 7.3440 & 0.1284 \\
0.14 & 9.4095 & 5.2420 & 7.8582 & 0.1443 \\
0.15 & 11.4909 & 6.9362 & 9.0924 & 0.1519 \\
0.16 & 16.9854 & 10.6156 & 9.7052 & 0.1680 \\
0.17 & 14.5023 & 9.7018 & 9.9428 & 0.1680 \\
0.18 & 71.4598 & 14.1405 & 12.8741 & 0.1797 \\
0.19 & 71.4814 & 9.5097 & 11.0223 & 0.1835 \\
0.20 & 18.2066 & 9.8277 & 10.8293 & 0.1890 \\
\hline
\end{tabular}

represents $n=400$ simulations of the lightning events for the fixed value of the azimuth $\theta$. For the sake of comparison of the estimators, for each value of $\theta, n$ lightning events were generated at the points $\left(x_{j}, y_{j}, z_{j}\right)$ with $x_{j}=D_{j} \cos \left(\alpha_{\jmath}\right)$, $y_{j}=D_{j} \sin \left(\alpha_{j}\right)$, and $z_{j}=D_{j} \tan \theta$, where the random horizontal distance from the origin $D_{\jmath}$ and the horizontal angle $\alpha$, were generated as uniform random variables in the intervals $(0,20 \mathrm{~km})$ and $(0,2 \pi)$, respectively. The first column of table 4 contains the value of the azimuth in degrees. The second, third, and fourth columns represent the values of the relative error $\delta$ of the LSE, MLE, and SSLE, respectively. The last column gives the estimators $\widehat{\sigma}$.

Table 5 represents simulation results when time measurements contain a systematic error. For the purpose of

Table 4. The Relative Error $\delta$ for Various Values of Azimuth $\theta$

\begin{tabular}{|c|c|c|c|c|}
\hline$\theta$, degrees & $\delta \mathrm{LSE}, \%$ & $\delta \mathrm{MLE}, \%$ & $\delta$ SSLE, $\%$ & $\widehat{\sigma}$ \\
\hline 2 & 7.53 & 7.08 & 8.56 & 0.0534 \\
\hline 4 & 8.35 & 8.83 & 11.05 & 0.0521 \\
\hline 6 & 6.84 & 6.33 & 8.17 & 00497 \\
\hline 8 & 7.48 & 7.10 & 9.43 & 0.0515 \\
\hline 10 & 7.01 & 6.57 & 8.32 & 0.0505 \\
\hline 12 & 6.80 & 6.41 & 7.98 & 0.0482 \\
\hline 14 & 6.30 & 5.85 & 6.96 & 0.0493 \\
\hline 16 & 5.96 & 5.51 & 6.56 & 0.0489 \\
\hline 18 & 6.06 & 5.45 & 6.46 & 0.0506 \\
\hline 20 & 5.14 & 4.81 & 5.58 & 0.0490 \\
\hline 22 & 9.44 & 9.40 & 10.27 & 0.0498 \\
\hline 24 & 5.29 & 4.83 & 5.55 & 0.0493 \\
\hline 26 & 4.71 & 4.22 & 5.13 & 0.0516 \\
\hline 28 & 4.52 & 4.15 & 4.70 & 0.0500 \\
\hline 30 & 4.62 & 4.17 & 4.74 & 0.0501 \\
\hline 32 & 4.67 & 4.37 & 4.80 & 0.0500 \\
\hline 34 & 3.94 & 3.58 & 4.06 & 0.0495 \\
\hline 36 & 3.91 & 3.61 & 4.19 & 0.0495 \\
\hline 38 & 4.36 & 5.10 & 14.03 & 0.0493 \\
\hline 40 & 3.62 & 3.38 & 3.72 & 0.0496 \\
\hline 42 & 4.36 & 4.16 & 4.70 & 0.0494 \\
\hline 44 & 4.50 & 4.19 & 4.55 & 0.0495 \\
\hline 46 & 3.70 & 3.39 & 3.70 & 0.0498 \\
\hline 48 & 4.21 & 3.81 & 4.20 & 0.0506 \\
\hline 50 & 4.50 & 4.28 & 4.48 & 0.0508 \\
\hline 52 & 3.74 & 3.49 & 3.68 & 0.0514 \\
\hline 54 & 4.08 & 3.84 & 4.13 & 0.0503 \\
\hline 56 & 4.30 & 4.10 & 4.38 & 0.0491 \\
\hline 58 & 3.97 & 3.66 & 3.98 & 0.0496 \\
\hline 60 & 5.27 & 5.02 & 5.41 & 0.0511 \\
\hline 62 & 3.95 & 3.60 & 3.97 & 0.0510 \\
\hline 64 & 4.71 & 4.47 & 4.74 & 0.0452 \\
\hline 66 & 3.99 & 3.75 & 3.98 & 0.0515 \\
\hline 68 & 4.24 & 3.96 & 4.22 & 0.0512 \\
\hline 70 & 4.64 & 4.19 & 4.60 & 0.0502 \\
\hline 72 & 5.28 & 5.08 & 5.26 & 0.0508 \\
\hline 74 & 5.29 & 4.88 & 5.29 & 0.0504 \\
\hline 76 & 5.78 & 5.26 & 5.72 & 0.0495 \\
\hline 78 & 6.53 & 6.25 & 6.43 & 0.0503 \\
\hline 80 & 8.42 & 7.70 & 10.78 & 0.0494 \\
\hline 82 & 9.61 & 8.87 & 9.26 & 0.0493 \\
\hline 84 & 12.13 & 11.10 & 11.56 & 0.0500 \\
\hline 86 & 21.59 & 19.18 & 16.28 & 0.0507 \\
\hline
\end{tabular}


Table 5. The Values of $\Delta$ in the Presence of Systematic Error

\begin{tabular}{ccccc}
\hline Error, km & $\Delta$ LSE, km & $\Delta$ MLE, km & $\Delta$ SSLE, km & $\widehat{\sigma}$ \\
\hline 0.1 & 9.2502 & 5.6487 & 6.0834 & 0.1029 \\
0.2 & 7.1947 & 4.3507 & 6.0009 & 0.1003 \\
0.3 & 7.4976 & 5.4041 & 6.5723 & 0.1001 \\
0.4 & 9.0459 & 5.2962 & 7.1594 & 0.1017 \\
0.5 & 9.3450 & 5.5530 & 6.5409 & 0.1029 \\
0.6 & 8.7158 & 5.3203 & 6.9562 & 0.1080 \\
0.7 & 10.6461 & 6.3489 & 7.9109 & 0.1044 \\
0.8 & 10.4741 & 6.3604 & 8.1494 & 0.1065 \\
0.9 & 11.6566 & 7.2806 & 8.4784 & 0.1077 \\
1.0 & 14.1132 & 6.7194 & 9.4468 & 0.1099 \\
1.1 & 12.6690 & 6.5499 & 9.0212 & 0.1074 \\
1.2 & 55.0841 & 7.0247 & 9.8991 & 0.1138 \\
1.3 & 16.3316 & 6.8601 & 9.7647 & 0.1167 \\
1.4 & 15.3773 & 7.3548 & 10.1731 & 0.1134 \\
1.5 & 16.8575 & 7.4090 & 10.3732 & 0.1195 \\
1.6 & 22.7233 & 7.9563 & 10.3513 & 0.1255 \\
1.7 & 19.0331 & 7.7149 & 11.2887 & 0.1242 \\
1.8 & 39.0366 & 8.1012 & 11.1726 & 0.1280 \\
1.9 & 60.9738 & 9.6425 & 12.2166 & 0.1315 \\
2.0 & 70.3597 & 10.5684 & 14.3139 & 0.1319 \\
\hline
\end{tabular}

Here $\sigma=0.1 \mathrm{~km}$.

investigation of the effect of a systematic error in measurements, all time measurements at site 0 were delayed by $\left[(k) 333.6 \times 10^{-9}\right]$ with $\mathrm{k}=1,2, \ldots, 20$, which corresponds to the error of $[(k) 0.1 \mathrm{~km}]$ in distance measurements, $k=1,2, . ., 20$. For each value of $\mathrm{k}$, we performed $n=400$ simulations of lightning events uniformly distributed in the parallelepiped $[-40,40] \times[-40,40] \times[0,20]$. The first column of Table 5 gives the values of the systematic error in distance measurements; the second, third and fourth columns show the values of $\delta$ of the LSE, MLE, and SSLE, respectively; and the last column contains $\widehat{\sigma}$.

Figures 1 and 2 show the graphs of the relative errors $\delta$ for the LSE, MLE, and SSLE methods; when $\sigma=0.05 \mathrm{~km}$ and $\sigma=0.01 \mathrm{~km}$, respectively. The $x$ axes represent the distance between the lightning location and the origin. Figure 3 demonstrates the same three graphs when lightning locations are uniformly distributed in the parallelepiped $[-40,40] \times[-40,40] \times[0,20]$, and the values of $\sigma$ vary. In all three figures, the curves of the relative errors for the LSE, MLE, and SSLE are sketched in thin, thick and dashed lines, respectively. Figure 4 indicates the average number of iterations required by MLE or LSE, when $\sigma=0.05 \mathrm{~km}$ (thin line) and $\sigma=0.01 \mathrm{~km}$ (dashed line), and lightning events occur at different distances from the origin. Since, in the course of simulations, both MLE and LSE methods involved just the same average number of iterations for minimization of functions (4) and (16), each curve in Figure 4 represents both the LSE and MLE techniques.

Tables $1-5$ as well as Figures 1-3 show that both the standard deviation $\Delta$ and the relative error $\delta$ grow with the increase of $\sigma$, the distance from the origin and the systematic error. The fluctuations of the graphs are due to the stochastic nature of the errors and would vanish if a larger number of simulations (say, $n=1000$ instead of $n=100$ ) were performed.

\section{Discussion}

Even a cursory glance at the tables and figures show that the MLE method usually gives better precision than the other two methods. Moreover, simulations show that the MLE always gives better precision than the LSE technique. Taking into account that both the MLE and LSE require the same number of iterations, i.e., essentially, the same computing time, the conclusion follows immediately that techniques based on minimization of function (4) should be abandoned.

Comparing the MLE and SSLE approaches, one can see that, except for the cases when the lightning event occurs at the far periphery (Table $2, D \geq 70 \mathrm{~km}$ ), when the precision of the time measurements $\varrho=\sigma / c$ is very poor (Table 3 , $\varrho>0.16 / c \simeq 534 \times 10^{-9} \mathrm{~s}$ ) or when the azimuth is large (Table $4, \theta=86^{\circ}$ ), the mean squared error of MLE is less than

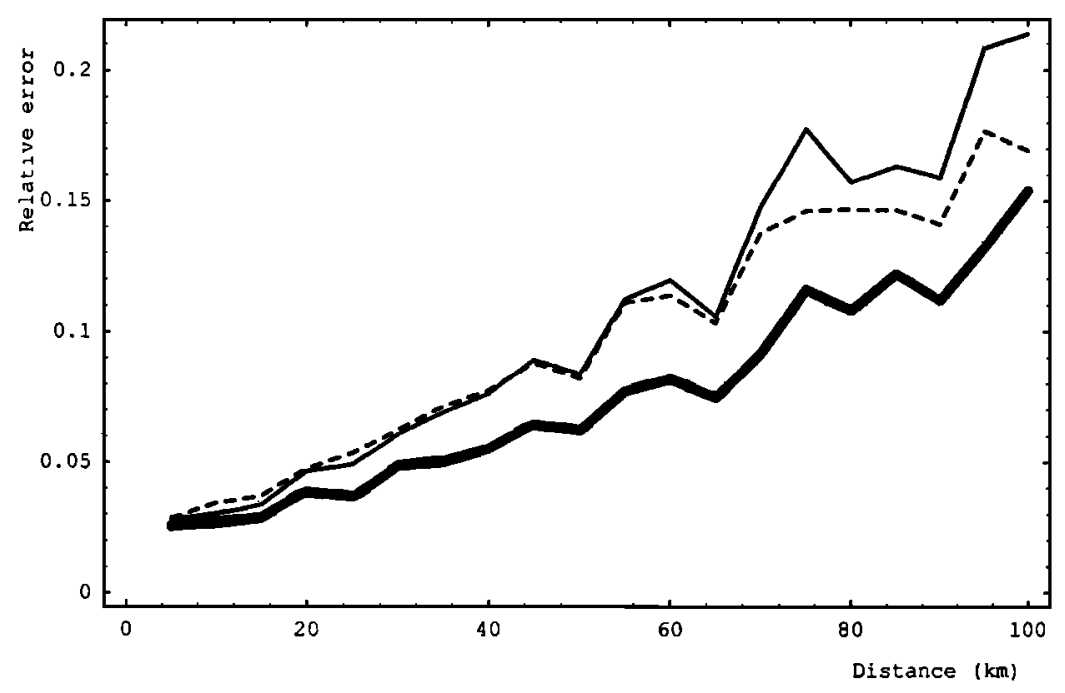

Figure 1. Relative errors of the LSE (thin line), MLE (thick line), and SSLE (dashed line) when the horizontal distance $D$ of the location of a lightning event from the origin varies. Standard deviation is $\sigma=0.05 \mathrm{~km}$. 


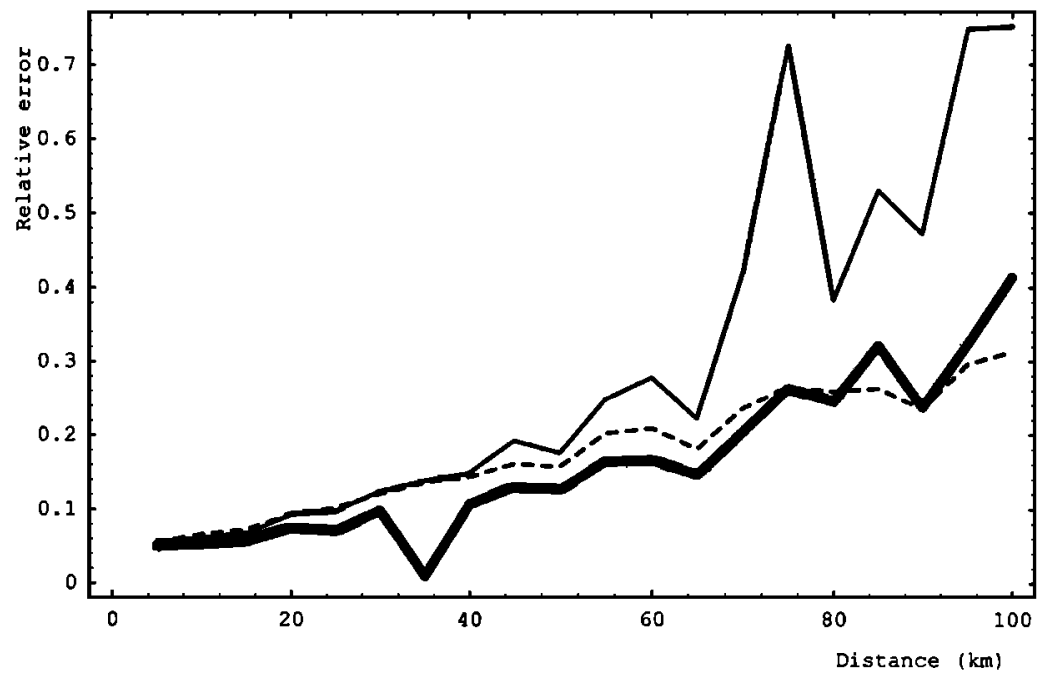

Figure 2. Relative errors of the LSE (thin line), MLE (thick line), and SSLE (dashed line) when the horizontal distance $D$ of the location of lightning event from the origin varies. Standard deviation is $\sigma=0.1 \mathrm{~km}$.

the mean squared error of SSLE. This means that if the time measurements are fairly accurate and a thunderstorm takes place in the neighborhood of the measuring sites, the MLE improves the results of SSLE. Moreover, Figures 1-3 show that unless a lightning event occurs very far away from the measuring sites (Figure 2, $D \geq 80 \mathrm{~km}$ ), the MLE gives smaller relative error than the other two methods. Note also that since the number of iterations required by MLE is relatively small, it does not lead to a significant increase of a computing time (see Figure 4). Furthermore, Table 5 demonstrates that the MLE is much less sensitive to the presence of a systematic error than the LSE and SSLE. Thus it is clearly the estimator of choice.

We draw attention to the fact that the MLE method does not discard the SSLE method but uses it as the initial iteration. Since the number of iterations required to reach the minimum increases and the precision of the method decays when the distance between the lightning location and the measuring sites grows, the MLE is not recommended for application if a thunderstorm occurs far from the area of interest (i.e., from the origin). When the thunderstorm occurs close to the origin (i.e. to the Kennedy Space Center, in our case) improvement of the precision of the estimation of lightning locations can be crucial to the safety of the work force.

The other merit of the MLE is that it provides the estimator $\widehat{\varrho}=\widehat{\sigma} / c$ of the precision of the time measurements $\varrho=\sigma / c$. This feature can be used for monitoring the measuring equipment as well as for evaluation of the precision of the estimators of lightning locations. A sudden increase of $\widehat{\varrho}$ indicates that a mistake was made while performing or recording time measurements. A steady growth of $\widehat{\varrho}$ signals that the equipment which implements the measurements should be checked. Besides that we want to emphasize that although all simula-

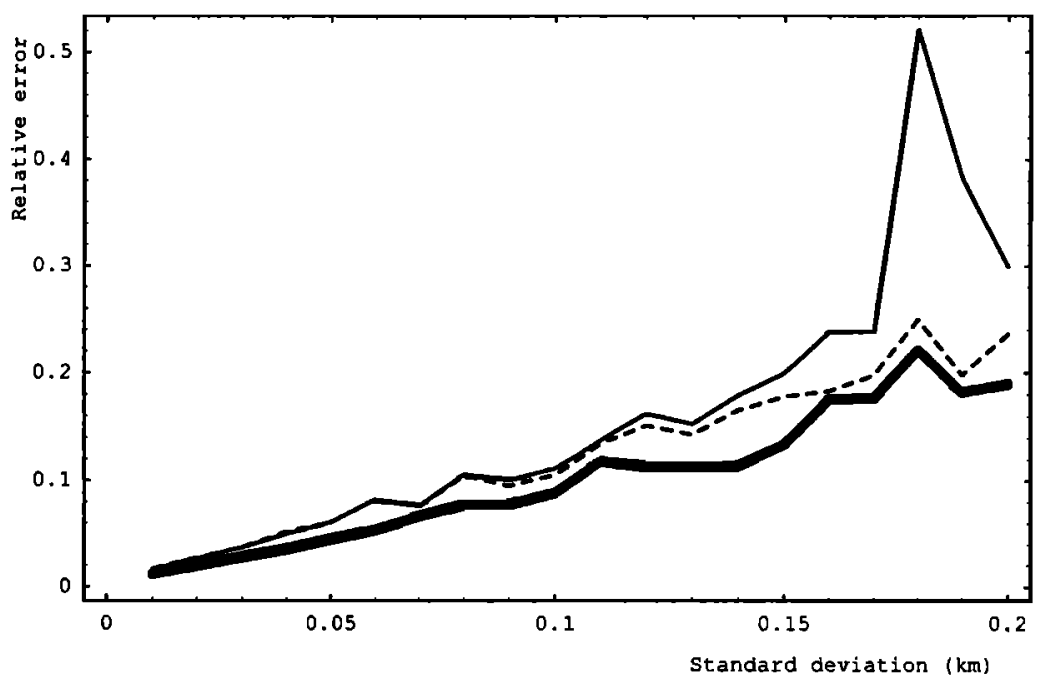

Figure 3. Relative errors of the LSE (thin line), MLE (thick line), and SSLE (dashed line) when the standard deviation $\sigma$ varies. The location of the lightning event is uniformly distributed in the parallelepiped $[-40 \mathrm{~km}, 40 \mathrm{~km}] \times[-40 \mathrm{~km}, 40 \mathrm{~km}] \times[0 \mathrm{~km}, 20 \mathrm{~km}]$. 


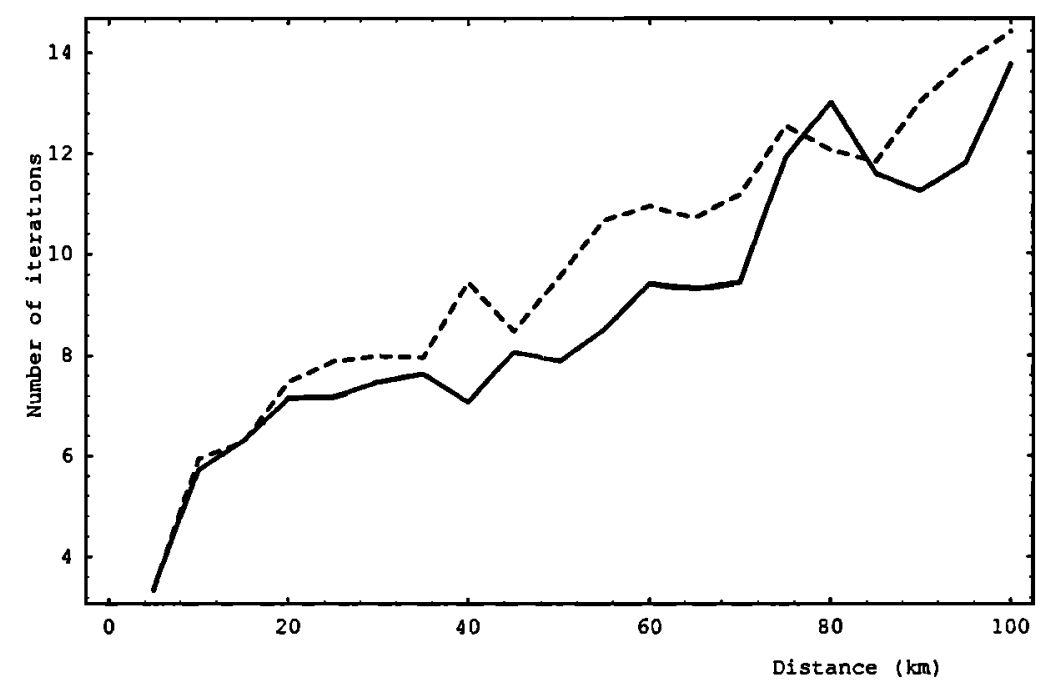

Figure 4. The averge number of iterations required by MLE or LSE, when the distance $D$ of the location of a lightning event from the origin varies and $\sigma=0.05 \mathrm{~km}$ (thin line) or $\sigma=0.01 \mathrm{~km}$ (dashed line).

tions were conducted in the case of ground-based measurements, the MLE technique can be applied without any changes in the general situation of arbitrarily located measuring sites.

It should also be recognized that the method of Monte Carlo simulations is very advantageous in evaluation and/or comparison of different techniques of retrieval of lightning locations. It is much simpler and cheaper than field experiments. The other merit is that the "true" location is always known, which makes examination of the precision of the methods more accurate. The Monte Carlo simulation method can be used for any number of measuring sites located arbitrarily in space. In fact, it can even be applied to investigate the best arrangement of the measuring sites.

\section{References}

Buslenko, N.P., D.I. Golenko, Y.A. Shreider, I.M. Sobol, and V.G. Sragovich, V.G., The Monte Carlo Method, Pergamon, Tarrytown, New York, 1966.

Cannon, J.R., A study of various methods for calculating locations of the lightning events, 1995 Research Report, NASA CR-199891, pp. 51-86, NASA/ASEE Summer Faculty Fellowship Prog., John F. Kennedy Space Cent. and Univ. of Cent. Fl., Orlando, Fl., 1995.

Cianos, N., G.N. Oetzel, and E.T. Pierce E.T., A technique for accurately locating lightning at close ranges, J. Appl. Meteorol., 11, 1120-1127, 1972.

Hager, W.H. and D. Wang, An analysis of errors in the location, current and the velocity of lightning, J. Geophys. Res., 100, 25,721-25,729, 1995.

Koshak, W.J. and R.J. Solakiewicz, On the retrieval of lightning radio sources from time-of-arrival data, J. Geophys. Res., 101, 26,631-26,639, 1996.

Lee, C.A., An experimental study of the remote location of lightning flashes using a VLF arrival time difference technique, $Q$. J. $R$. Meteorol. Soc, 112, 203-229, 1986.
Lehmann, E.L., Theory of Point Estimation, Wiley, 1983.

Lewis, E.A., R.B. Harvey, and J.E. Rasmussen, Hyperbolic direction finding with spherics od transatlantic origin, J. Geophys. Res., 65, 1879-1905, 1960.

MacClement, W.D., and R.C. Murty, VHF direction finder studies of lightning, J. Appl. Meteorol., 17, 786-795, 1978.

Murty, R.C., and W.D. MacClement, VHF direction finder for lightning location, J. Appl. Meteorol., 12, 1401-1405, 1973.

Panyukov, A.V., and V.A. Strauss, A method to determine parameters of a linear functional equations set and its applica-tion to the lightning location problem, in Parameter Identification and Inverse Problems in Hydrology, Geology and Ecology, edited by J. Gottlieb, and P. DuChateaua, pp. 199-209, Kluwer Acad., New York, 1996.

Press, W.H., B.P. Flannery, S.A. Teukolsky, W.T. Vettering, Numerical Recipes, The Art of Scientific Computing, Cambridge Univ. Press, New York, 1986.

Proctor, D.E., A hyperbolic system for obtaining VHF radio pictures of lightning, J. Geophys. Res., 76, 1478-1489, 1971.

Rustan, P.L., M.A. Uman, D.G. Childers, W.H. Beasley, and C.L. Lennon, Lightning source locations from VHF radiation data for a flash at Kennedy Space Center, J. Geophys. Res., 85, 4893-4903, 1980.

Taylor, W.L., A VHF technique for space-time mapping of lightning discharge processes, J. Geophys. Res., 83, 3575- 3583, 1978.

Thompson, E.M., P.J. Medelius, and S. Davis, A system for locating the sources of wideband $\mathrm{dE} / \mathrm{dt}$ from lightning, J. Geophys. Res., 99, 22,793-22,802, 1994.

J. R. Cannon and M. Y. Pensky, Department of Mathematics, University of Central Florida, University of Central Florida, Orlando FL 32816. (jcannon@pegasus.cc.ucf.edu; mpensky@pegasus.cc.ucf.edu)

(Received March 9, 1998; revised July 22, 1998; accepted November 27, 1998.) 